\title{
Comparación de técnicas quirúrgicas para la lesión del manguito de los rotadores: costo-resultado
}

\section{Comparison of surgical techniques for rotator cuff injury: cost-result}

\author{
Velasco-Alcázar R,* Rojas-Sosa MC ${ }^{\star}$ Navarro-López MG, ${ }^{\S}$ Ibáñez-Galicia M, \\ Chávez-Covarrubias G, Olvera-Gómez JL, ** Pérez-Atanasio JM*
}

Hospital General Regional No. 2 «Villa Coapa», IMSS.

RESUMEN. Introducción: Actualmente, para manejar la ruptura del manguito de los rotadores (LMR), se prefiere la artroscopía sobre técnicas abiertas (mini-open [MO]), con resultados y costos controversiales. Objetivo: Analizar el costo-resultado de las técnicas quirúrgicas para la LMR. Material y métodos: Se realizó una evaluación económica parcial de tipo costo-resultado a 113 pacientes con LMR intervenidos mediante técnica miniopen $(\mathrm{n}=6)$ o artroscopía $(\mathrm{n}=91)$ o ambas $(\mathrm{n}=16)$. Los costos directos incluyeron: estancia, días de incapacidad laboral, cirugía, implantes (anclas), valoraciones médicas, laboratorio e imagen. Se evaluó la funcionalidad del hombro a un año mediante la escala de Constant-Murley (EscC-M). Resultados: La técnica MO fue la más económica $(\mathrm{X}=\$ 42,950.60 \mathrm{MXN})$ y la más costosa fue la artroscopía + MO con anclas $(\mathrm{X}=\$ 89,594.80 \mathrm{MXN})$; con una diferencia no significativa en la funcionalidad del hombro (EscC-M = 56.33 \pm 7 vs $64.25 \pm 9$ puntos, respectivamente) ( $\mathrm{p}>0.05$; Kruskal-Wallis; post hoc Mann-Whitney). Una diferencia de $\$ 46,644.10 \mathrm{MXN}$ entre técnicas correspondió a 7.92 puntos. Hubo relación leve entre la técnica y la funcionalidad del hombro $\left(\mathrm{r}_{\mathrm{s}}=\right.$ $0.26 ; \mathrm{p}=0.005$; Spearman). Conclusión: La técnica $m i$ $n i$-open mostró mejor costo-resultado, con una ganancia
ABSTRACT. Introduction: Currently, to manage rotator cuff injury (RCI), there is a tendency to replace mini-open (MO) surgery with arthroscopy, but results and costs are controversial. Objective: To analyze the cost-result of surgical techniques for RCI. Material and methods: A partial economic evaluation of cost-result type was performed on 113 patients with RCI surgically operated by mini-open technique $(n=6)$, or arthroscopy $(n=91)$, or both $(n=16)$. Direct costs included: hospital stay, days of work disability, surgery, implants (anchors), medical assessments, laboratory and imaging. One-year shoulder functionality was evaluated with ConstantMurley scale (EscC-M). Results: The MO technique was the most economical $(\mathrm{X}=\$ 42,950.60 \mathrm{MXN})$ and the most expensive was the arthroscopy + MO with anchors ( $\mathrm{X}=\$ 89,594.80 \mathrm{MXN})$; with a non-significant difference in shoulder functionality (EscC-M = 56.33 \pm 7.0 vs. $64.25 \pm 9.0$ points, respectively) $(\mathrm{p}>0.05$ Kruskal-Wallis; post hoc Mann-Whitney). Difference of $\$ 46,644.1 \mathrm{MXN}$ between techniques corresponded to 7.92 points. There was a mild relationship between the technique and the functionality of shoulder $\left(\mathrm{r}_{\mathrm{s}}=0.26 ; \mathrm{p}=\right.$ 0.005; Spearman). Conclusion: The mini-open technique showed best cost-result, with similar functionality

\section{Nivel de evidencia: III}

* Servicio de Extremidad Torácica, Hospital General Regional No. 2 «Villa Coapa», Instituto Mexicano del Seguro Social (IMSS). Ciudad de México, México.

* Coordinación de Planeación y Enlace Institucional, Delegación Sur del Distrito Federal, IMSS. Ciudad de México, México.

§ Servicio de Urgencias, Hospital General Regional No. 2 «Villa Coapa», IMSS. Ciudad de México, México.

" Servicio de Extremidad Pélvica I, Hospital General Regional No. 2 «Villa Coapa», IMSS. Ciudad de México, México.

" Dirección, Hospital General Regional No. 2 «Villa Coapa», IMSS. Ciudad de México, México.

** Titular de la Coordinación de Planeación y Enlace Institucional, Delegación Sur, IMSS. Ciudad de México, México.

" Servicio de Reemplazo Articular, Hospital General Regional No. 2 «Villa Coapa», IMSS. Ciudad de México, México.

Correspondencia:

María del Carmen Rojas-Sosa

Av. Durango Núm. 289, piso 4a, Col. Roma Norte, CP 06700, Alcaldía Cuauhtémoc, Ciudad de México.

E-mail: mc_rojass@yahoo.com.mx

Citar como: Velasco-Alcázar R, Rojas-Sosa MC, Navarro-López MG, Ibáñez-Galicia M, Chávez-Covarrubias G, Olvera-Gómez JL et al. Comparación de técnicas quirúrgicas para la lesión del manguito de los rotadores: costo-resultado. Acta Ortop Mex. 2020; 34(5): 282-287. https://dx.doi.org/10.35366/97989

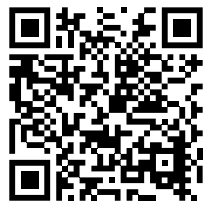


en la funcionalidad de hombro similar a la que se obtiene con la técnica más costosa.

Palabras clave: Manguito de los rotadores, análisis económico, artroscopía, técnica mini-open.

\section{Introducción}

Las enfermedades del manguito de los rotadores son causa principal de consulta por hombro doloroso a nivel mundial. ${ }^{1}$ Desde hace cuatro décadas, el número de artroscopías para reparar lesiones del manguito de los rotadores (LMR) ha ido en aumento, debido a que la incisión era más pequeña y producía menor dolor postoperatorio. ${ }^{2}$ No obstante, existe evidencia variable al considerar la artroscopía sobre los procedimientos abiertos (MO) para manejar las LMR en diversos países. ${ }^{3,4}$ La literatura mostró controversia en el dolor postquirúrgico, ${ }^{5,6}$ sin diferencias significativas en tiempos de cirugía, resultados funcionales y rango de movimiento entre las técnicas, ${ }^{6}$ con mejor preservación de estructuras del hombro con la MO. ${ }^{2}$

Pocos artículos han publicado los costos quirúrgicos de la LMR. Inglaterra reportó un costo unitario de $£ 6,628^{7}$ y Singapur entre \$5,027.06-\$11,542.33 SGD/paciente. ${ }^{8}$ En general, no se encontró literatura reciente del gasto en LMR, algunos investigadores proporcionan información limitada sobre los factores incluidos en los costos quirúrgicos ${ }^{9} \mathrm{o}$ sólo un estimado directo global, ${ }^{10}$ cuando en otros países sí consideraron consumibles e implantes. ${ }^{10,11}$

La escala de Constant-Murley (EscC-M) ha sido usada en múltiples publicaciones del campo de la ortopedia, ${ }^{3,8,12,13}$ pues evalúa subjetiva y objetivamente la función de la extremidad superior y sus resultados son similares a las escalas de DASH y Oxford Shoulder Score ${ }^{13}$ es genérica, sencilla y aplicable, prácticamente, en todas las patologías de hombro. ${ }^{14}$

No se encontró literatura reciente de costos de la cirugía para LMR, en México. En el Hospital General Regional No. 2 , también ha disminuido el uso de la $\mathrm{MO},{ }^{2}$ pero con tendencia de iniciar con artroscopía y concluir con MO.

El objetivo planteado fue analizar los costos de las técnicas MO y artroscópica en el manejo de las LMR y compararlos con el resultado funcional del hombro un año después.

\section{Material y métodos}

Se realizó una evaluación económica parcial de tipo costo-resultado, con perspectiva institucional, en expedientes de pacientes con LMR, intervenidos quirúrgicamente en el HGR2VC, mediante técnica MO sola o artroscópica con y sin anclas o una combinación de ambas (mixta) con y sin anclas, entre enero de 2016 a diciembre de 2017. Solamente se incluyeron expedientes con información completa: nom- in shoulder to that obtained with the more expensive technique.

Keywords: Rotator cuff injury, economic analysis, arthroscopy, mini-open technique.

bre, edad, sexo, diagnóstico, cirugía realizada, técnica quirúrgica, consultas de especialidad, días de estancia intrahospitalaria, días de incapacidad temporal para el trabajo (ITT), rehabilitación, estudios prequirúrgicos, procedimientos y con evaluación funcional del hombro mediante la EscC-M.

\section{Mediciones y costeo de las intervenciones quirúrgicas}

Para detallar mejor los costos, las técnicas quirúrgicas se dividieron en cinco grupos: 1. MO solamente (MO), 2. Artroscopía más MO sin anclas (Artrosc + MOs/anc), 3. Artroscopía más MO con anclas (Artrosc + MOc/anc), 4. Artroscopía sola sin anclas (ArtroscS/anc) y 5. Artroscopía sola con anclas (ArtroscC/anc).

El costo directo de las cinco técnicas incluyó: número de consultas en el servicio de extremidad torácica, valoraciones prequirúrgicas (anestésica, medicina interna, etc.), consultas de especialidad, curaciones, laboratorio, electrocardiograma y telerradiografía de tórax, resonancia magnética, ultrasonido o tomografía computarizada, rehabilitación y días de ITT. Los costos quirúrgicos se obtuvieron del reporte mensual de bienes de consumo y de los costos unitarios de segundo nivel de atención médica del 2017 en el IMSS (Tabla 1); el costo de los días de ITT otorgados se obtuvo del Sistema de Incapacidad Nacional, ambos en pesos mexicanos $(\mathrm{MXN}){ }^{15}$

Efectividad. La medida de resultado fue la funcionalidad del hombro evaluada con la EscC-M, a un año de la cirugía. La escala incluye: medición del dolor (15 puntos), movilidad de vida diaria (20 puntos: trabajo, deporte, sueño, amplitud de movimiento indoloro), movilidad activa (40 puntos: abducción, flexión y rotación externa e interna) y potencia ( 25 puntos); la puntuación máxima alcanzable son 100 puntos. La funcionalidad se consideró excelente cuando se obtuvieron 80 puntos o más, buena entre 65 a 79 , media con 50 a 64 y malo $<50$ puntos. ${ }^{14}$

Métodos estadísticos. La distribución de costos y puntuaciones fue no normal (Kolmogórov-Smirnov), por lo que se utilizó estadística no paramétrica. Se utilizaron las pruebas de Kruskal-Wallis (post hoc Mann-Whitney), $\chi^{2}$ y el coeficiente de correlación de Spearman. Se consideró significativa una $\mathrm{p} \leq 0.05$.

Aspectos éticos. Este trabajo fue aceptado por un comité local de investigación del IMSS, considerado como investigación sin riesgo, los individuos involucrados otorgaron un consentimiento informado para participar en el estudio, de acuerdo al Reglamento en Materia de Investigación. ${ }^{16}$ 
Tabla 1: Costo unitario por procedimiento, reporte mensual de bienes de consumo de segundo nivel de atención médica en el IMSS, valores en pesos mexicanos del año 2017. ${ }^{20}$

\begin{tabular}{|lc|}
\hline Procedimientos & $\begin{array}{c}\text { Costo (pesos } \\
\text { mexicanos }[\mathrm{MXN}])\end{array}$ \\
\hline Técnica abierta & $\$ 19,327.0$ \\
Técnica artroscópica & $\$ 25,625.0$ \\
Ancla & $\$ 8,569.0$ \\
Día estancia intrahospitalaria & $\$ 7,256.0$ \\
Terapia de rehabilitación & $\$ 1,152.0$ \\
Valoración por anestesiología & $\$ 1,001.0$ \\
Valoración medicina interna & $\$ 1,001.0$ \\
Consulta ortopedia & $\$ 1,001.0$ \\
Curaciones & $\$ 674.0$ \\
Electrocardiograma & $\$ 319.0$ \\
Laboratorios & $\$ 102.0$ \\
Telerradiografía de tórax & $\$ 283.0$ \\
Ultrasonido & $\$ 487.0$ \\
Resonancia magnética nuclear & $\$ 4,341.0$ \\
Tomografía computada & $\$ 2,846.0$
\end{tabular}

\section{Resultados}

Se revisaron 184 expedientes de pacientes intervenidos quirúrgicamente por LMR, pero solamente 113 cumplieron con los criterios de selección. De los 113 sujetos, 36 fueron hombres (32\%) y 77 mujeres $(68 \%)$, con edad $\mathrm{X}=57.42 \pm$ 13.75 años. Hubo seis pacientes (5.3\%) intervenidos con la técnica $\mathrm{MO}, 91$ pacientes $(80.5 \%)$ con artroscopía, con o sin anclas y $16(14.2 \%)$ que iniciaron con artroscopía y continuó con MO (mixta), con o sin anclas.

Aunque la edad no fue significativamente diferente entre los 5 grupos ( $\mathrm{p}=0.11$; Kruskal-Wallis $)$, si hubo significancia entre la edad de MO $(\mathrm{X}=64 \pm 3.03$ años $)$ con Artrosc + MOs/anc ( $\mathrm{X}=48 \pm 9.84$ años) y con ArtroscC/anc $(X=52$ \pm 17.7 años) ( $p<0.05$; post hoc Mann-Whitney). Se identificó una mayor proporción de mujeres intervenidas quirúrgicamente, comparadas con los hombres $(68 \%$ vs $32 \%$, respectivamente; razón $2.1 / 1)\left(\mathrm{p}<0.05 ; \chi^{2}\right)$. Considerando la puntuación alcanzada con la EscC-M, 35.4\% tuvo una funcionalidad de hombro mala, $63.63 \%$ tuvo una funcionalidad media a buena y solamente un paciente tuvo un resultado excelente $(0.88 \%)$. El costo total por el manejo quirúrgico de los 113 sujetos fue de \$6’445,337.20 MXN (Tabla 2).

El análisis de sensibilidad mostró que la cirugía MO fue la más económica de todas $(\$ 42,950.60 \mathrm{MXN} /$ paciente) y la más costosa fue iniciar con artroscopía, continuar con abierta y utilizar anclas $(\$ 89,594.80 \mathrm{MXN} /$ paciente). Al comparar las puntuaciones (EscC-M) obtenidas con estas técnicas, las dos correspondieron a un nivel medio de funcionalidad de hombro (56.33 vs 64.25 puntos, respectivamente). La diferencia entre ambas cirugías fueron 7.92 puntos/promedio y $\$ 46,644.10 \mathrm{MXN} /$ promedio ( $<<0.05$; Kruskal-Wallis; $\mathrm{p}$ $=0.07$, post hoc Mann-Whitney). La puntuación más baja correspondió a la artroscopía $+\mathrm{MOs} /$ anc $(\mathrm{X}=40.0 \pm 4.0$ puntos; costo $\$ 76,865.90 \pm \$ 14,543.10 \mathrm{MXN}$ ) y la más

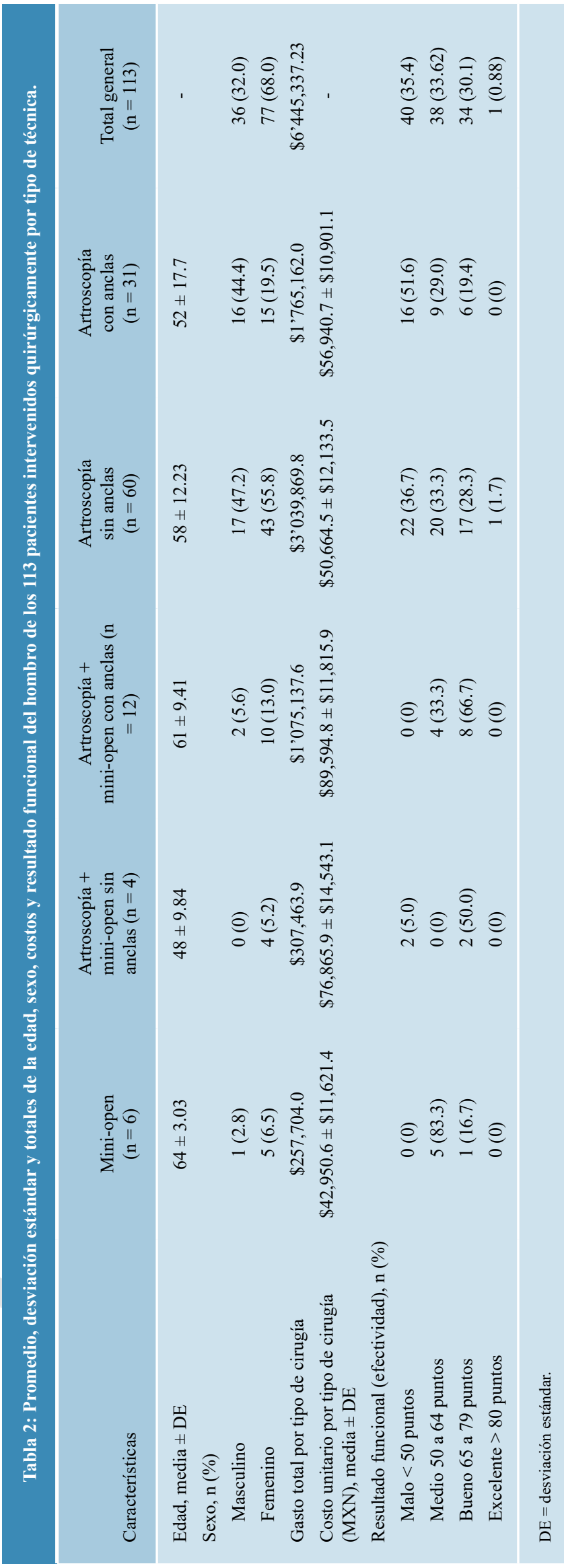


alta en la artroscopía + MOc/anc (64.25 \pm 9.0 puntos; costo $\$ 89,594.80 \pm \$ 11,815.90 \mathrm{MXN})$, la diferencia entre ambas cirugías fue de 24.25 puntos/promedio y $\$ 12,728.80 \mathrm{MXN}$ (p $<0.05$; Kruskal-Wallis; post hoc Mann-Whitney) (Tabla 3).

Se documentó una relación leve entre el tipo de técnica quirúrgica con el resultado funcional $\left(\mathrm{r}_{\mathrm{s}}=0.26 ; \mathrm{p}=0.005\right.$; Spearman). Se observó una tendencia inversa entre el costo de la técnica quirúrgica con la funcionalidad del hombro (EscC-M), ya que a menor costo se obtuvo mayor puntuación promedio en las técnicas mini-open y artroscópica sola, al compararlas con las técnicas más caras (Figura 1).

Hubo 27 sujetos que recibieron 2,997 días de ITT, con un costo total de $\$ 1 ’ 359,219.10 \mathrm{MXN}$. Cuando se agregó el costo por incapacidad al gasto quirúrgico, la técnica más cara por paciente fue la Artrosc + MOc/anc $(\$ 139,269.40 \pm$ $48,795.10 \mathrm{MXN}$ ) y la más económica fue la ArtroscC/anc $(\$ 76,788.60 \pm 20,273.70 \mathrm{MXN})$, que también fue la que se asoció a un menor número de días de incapacidad (Tabla 4). La cirugía con mayor cantidad de días de incapacidad por paciente fue la Artrosc $+\mathrm{MOs} /$ anc. El gasto quirúrgico más incapacidad en estos 27 pacientes fue de \$2’949,580.90 MXN. La inversión del IMSS al agregar los costos por la ITT fue de \$7’804,556.40 MXN.

\section{Escenarios económicos}

Escenario 1: cirugía con la técnica más económica en los 113 pacientes; la inversión institucional habría sido \$4'853,425.70 MXN, con ahorro de \$1'591,911.50 MXN.

Escenario 2: cirugía con la técnica más cara en los 113 pacientes; el costo total habría sido de $\$ 10^{\prime} 124,212.40$ MXN.

En los dos escenarios, para obtener un resultado funcional medio del hombro.

Escenario 3: cirugía de los 184 pacientes; manejados con la opción menos costosa, gasto de: \$7'902,923.20 MXN o con la más cara: $\$ 16^{\prime} 485,443.20 \mathrm{MXN}$.

Si en cada escenario se agrega $\$ 1^{\prime} 359,219.10 \mathrm{MXN}$, por la ITT, el espectro del gasto institucional podría ubicarse entre \$6’212,644.90 MXN y \$17’844,662.30 MXN, en el período estudiado.

\section{Discusión}

La controversia persiste, algunos prefieren la artroscopía para manejar las LMR, por necesitar una incisión pequeña y producir menos dolor; con tendencia a abandonar las técnicas «abiertas», como la mini-open (MO) ${ }^{2,8} \mathrm{Al}$ margen de las ventajas y desventajas de ambas técnicas, ${ }^{17}$ se ha descrito que la MO ha mostrado un mayor costo-resultado compara-

Relación entre el costo quirúrgico y la puntuación funcional del hombro (promedios)

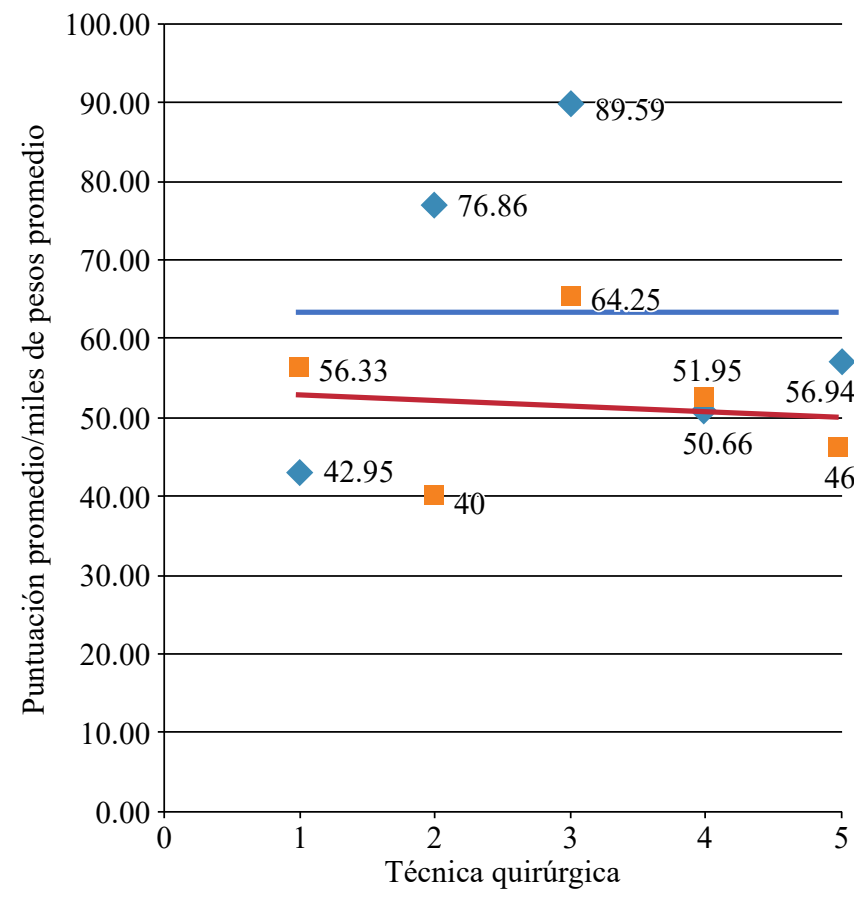
Costo (miles)
- Efectividad (puntos)
_ Lineal (costo [miles])
Lineal (efectividad [puntos])

1 = mini-open; 2 = artroscopía + mini-open sin anclas; 3 = artroscopía + mini-open con anclas; 4 = artroscopía sin anclas; 5 = artroscopía con anclas.

Figura 1: Comparación entre el costo promedio y la puntuación promedio de la funcionalidad postquirúrgica de hombro obtenida con la escala de Constant-Murley por técnica quirúrgica.

Tabla 3: Comparación del costo por intervención quirúrgica unitaria promedio, según la técnica utilizada, con la funcionalidad del hombro medida en puntos obtenidos con la escala de Constant-Murley en los $\mathbf{1 1 3}$ pacientes con lesión del manguito de los rotadores.

\begin{tabular}{|c|c|c|c|c|}
\hline \multirow[b]{2}{*}{ Técnica } & \multicolumn{2}{|c|}{ Costos promedio por cirugía unitaria $\left(\mathrm{MXN}^{*}\right)$} & \multicolumn{2}{|c|}{$\begin{array}{c}\text { Efectividad } \\
\text { Escala de Constant-Murley (puntos) }\end{array}$} \\
\hline & Media \pm DE & Rango & Media $\pm \mathrm{DE}$ & Rango \\
\hline Mini-open $(\mathrm{n}=6)$ & $42,950.6 \pm 11,621.4$ & $32,554.0-62,716.0$ & $56.33 \pm 7$ & $50-65$ \\
\hline Artroscopía más mini-open $\sin$ anclas $(n=4)$ & $76,865.9 \pm 14,543.1$ & $62,604.2-96,974.2$ & $40.0 \pm 4.0$ & $4.0-78$ \\
\hline Artroscopía más mini-open con anclas $(\mathrm{n}=12)$ & $89,594.8 \pm 11,815.9$ & $70,801.9-110,696.9$ & $64.25 \pm 9.0$ & $51-78$ \\
\hline Artroscopía sin anclas $(n=60)$ & $50,664.5 \pm 12,133.5$ & $38,299.2-97,062.4$ & $51.95 \pm 17$ & $21-81$ \\
\hline Artroscopía con anclas $(\mathrm{n}=31)$ & $56,940.7 \pm 10,901.1$ & $45,229.9-79,967.9$ & $46 \pm 17$ & $10-78$ \\
\hline
\end{tabular}




\begin{tabular}{|c|c|c|c|c|c|c|}
\hline Tipo de cirugía & $\mathrm{n}$ & $\begin{array}{l}\text { Días de incapacidad } \\
\text { total }(\text { media } \pm \mathrm{DE})\end{array}$ & $\begin{array}{c}\text { Costo } \\
\text { incapacidad* } \\
\text { (total) }\end{array}$ & $\begin{array}{l}\text { Costo cirugía } \\
\text { (total) }\end{array}$ & $\begin{array}{l}\text { Costo final total } \\
\text { (incapacidad + } \\
\text { cirugía) }\end{array}$ & $\begin{array}{l}\text { Costo total promedio } \\
\quad(\text { media } \pm \mathrm{DE})\end{array}$ \\
\hline Artroscopía + MO sin anclas & 3 & $\begin{array}{c}647 \\
215.6 \pm 174.4\end{array}$ & $95,688.4$ & $244,859.7$ & $340,548.1$ & $113,516.0 \pm 28690.3$ \\
\hline Artroscopía + MO con anclas & 2 & $\begin{array}{c}280 \\
140 \pm 67.8\end{array}$ & $104,290.9$ & $174,247.9$ & $278,538.8$ & $139,269.4 \pm 48,795.1$ \\
\hline Artroscopía sin anclas & 14 & $\begin{array}{c}1,656 \\
118.3 \pm 114.3\end{array}$ & $1^{\prime} 012,899.6$ & $703,285.3$ & $1^{\prime} 716,184.9$ & $122,584.6 \pm 133,400.4$ \\
\hline Artroscopía con anclas & 8 & $\begin{array}{c}414 \\
51.75 \pm 66.4\end{array}$ & $146,340.2$ & $467,968.7$ & $614,308.9$ & $76,788.6 \pm 20,273.7$ \\
\hline Total & 27 & 2,997 & $1^{\prime} 359,219.1$ & $1^{\prime} 590,361.7$ & $2^{\prime} 949,580.9$ & - \\
\hline
\end{tabular}

da con la artroscopía, sin diferencia en la funcionalidad del hombro y dolor, $6,8,11,17,18$ similar a los hallazgos en el presente estudio, en donde se encontró que la MO tuvo el menor costo al compararla con las otras cuatro formas quirúrgicas (Tabla 2) y con un resultado funcional medio del hombro (EscC-M), similar a la técnica más costosa $(\mathrm{X}=56.33$ vs 64.25 puntos, respectivamente), a largo plazo (12 meses); lo que la vuelve la cirugía más rentable. ${ }^{11}$ La diferencia de 7.9 puntos entre la cirugía más económica $(\mathrm{MO})$ y la más cara (Artrosc $+\mathrm{MOc} / \mathrm{anc})$, fue no significativa $(\mathrm{p}>0.05$; Kruskal-Wallis; post hoc Mann-Whitney) y correspondió a $\$ 46,644.10$ pesos de diferencia entre ambas. Se documentó una relación leve $\left(\mathrm{r}_{\mathrm{s}}=0.26 ; \mathrm{p}=0.005\right.$; Spearman) entre el costo de las cirugías con los resultados clínicos (funcionalidad de hombro con EscC-M), encontrando una mejor puntuación ante cirugías de menor costo (Figura 1).

Aunque el costo unitario de la cirugía para la LMR en el HGR2VC es bajo, comparado con otros países, ${ }^{8,7,11}$ no se halló literatura reciente de México sobre este gasto. El costo total directo de la artroscopía está más relacionado con el material utilizado, como las anclas, ${ }^{10,11}$ ya que no se afecta por el paciente, características de la lesión, la técnica o tiempo quirúrgico. ${ }^{9} \mathrm{La} \mathrm{MO}$ parece realizarse en un menor lapso versus la artroscopía, ${ }^{9,10}$ lo que influye en la disminución de costos; aunque los tiempos podrían variar dependiendo del tipo de lesión y la experiencia y formación del cirujano. ${ }^{19,20}$

Existen algunos trabajos donde los costos y resultados clínicos de la artroscopía o reparación abierta no tuvieron diferencia significativa ${ }^{11,17,18,19}$, algunos no describen lo que incluyeron en los procedimientos; ${ }^{7,17}$ por lo que las controversias de costos y resultados de la artroscopía (sola o mixta) y la cirugía abierta pueden relacionarse con los gastos incluidos. Al igual que otros autores, en este estudio se incluyeron los costos prequirúrgicos, transquirúrgicos y postquirúrgicos, al comparar las diferentes técnicas, con resultados similares. ${ }^{11}$

Se ha descrito que se prefiere manejar con técnica $\mathrm{MO}$ a pacientes de 50 años o más, ${ }^{17} \mathrm{o}$ incluso en adultos mayores $(+65$ años $),{ }^{21}$ y aquí se encontró que el mayor grupo etario se ubicó en la técnica MO $(X=64 \pm 3.03$ años $)$, seguido de la Artrosc + MOc/anc ( $\mathrm{X}=61 \pm 9.41$ años $)$; aunque la edad no es criterio quirúrgico del HGR2VC al seleccionar el tipo de intervención. Tampoco se han reportado diferencias significativas entre géneros, ${ }^{21}$ pero aquí, dentro de la cohorte se encontró una proporción mujer/hombre de 2.1 a 1 .

En estudios de costo-efectividad previos, se encontró que la simple intervención quirúrgica de las LMR mejora la calidad de vida, ${ }^{22}$ sin diferencia significativa en la efectividad valorada por escalas, ${ }^{19,23}$ ni en los costos. ${ }^{22}$ En este trabajo no se midió la calidad de vida, pero se identificó que $64.6 \%$ tuvieron una funcionalidad media a excelente del hombro a 12 meses de seguimiento (Tabla 2), lo que permitiría una mayor independencia para las actividades de la vida diaria.

También se encontró que los sujetos manejados con MO no generaron incapacidad, pero hubo 27 pacientes en las otras cuatro técnicas que ameritaron 2,997 días de ITT. Es importante resaltar que cuando se combinó la artroscopía con MO, los días/ITT fueron mayores que con la artroscopía sola (Tabla 4). Se ha publicado que no hay diferencias en la incapacidad entre MO y artroscopía, ${ }^{11}$ aunque con artroscopía los pacientes regresaron a trabajar más rápido. ${ }^{20}$ Los pacientes manejados con la artroscopía sola regresaron a laborar entre los 1.72 a 3.94 meses después de la cirugía, similar o menor a lo descrito por otros investigadores. ${ }^{18,24}$

Es interesante que la tendencia de usar más la artroscopía parece ser mundial, con un incremento de $188 \%$ en EU, de las que $68 \%$ fueron artroscopías y $32 \%$ mini-open. ${ }^{21}$ Acorde con esta tendencia, en dos años, en este estudio se encontró una proporción de $80.54 \%$ de artroscopías vs 5.3\% MO; el restante $14.16 \%$ se manejó iniciando con artroscopía y terminando con $\mathrm{MO}$, lo que aumentó el gasto en cada intervención (Tabla 2).

Hace aproximadamente 20 años, a manera de entrenamiento, se iniciaba con artroscopía para tener una excelente visión de la lesión y preparar el terreno para después realizar el anclaje del manguito rotador a cielo abierto, proponiendo que en el futuro se podría llegar a una solución totalmente endoscópica. ${ }^{25}$ Esto no ha sucedido en el HGR2VC y la evi- 
dencia que se tiene en la práctica sostiene que la selección de la técnica se debe a los hallazgos durante la intervención y un criterio personal del cirujano, quien inicia con una técnica y concluye con otra, pero esto puede ser considerado una debilidad y ser motivo de otro estudio.

\section{Conclusión}

El análisis presentado muestra que en el HGR2VC la cirugía MO tuvo el mayor costo-resultado en el manejo de las LMR, sin diferencia significativa a 12 meses en la funcionalidad del hombro, comparada con la técnica más cara (Artrosc $+\mathrm{MOc} / \mathrm{anc}$ ), con diferencia de 7.9 puntos y de $\$ 46,644.13 \mathrm{MXN}$ promedio entre ellas. Al comparar la técnica $\mathrm{MO}$ con la artroscopía sola, también mostró un mejor costo-resultado, con diferencia entre 4.38-10.33 puntos y de \$7,713.90-13,990.10 MXN. Encontrándose una tendencia de mejor resultado funcional a menor costo.

Referencias

1. Piper CC, Hughes AJ, Ma Y, Wang H, Neviaser AS. Operative versus nonoperative treatment for the management of full-thickness rotator cuff tears: a systematic review and meta-analysis. $J$ Shoulder Elbow Surg. 2018; 27(3): 572-6.

2. Levy HJ, Uribe JW, Delaney LG. Arthroscopic assisted rotator cuff repair: preliminary results. Arthroscopy. 1990; 6(1): 55-60.

3. Vitale MA, Vitale MG, Zivin JG, Braman JP, Bigliani LU, Flatow EL. Rotator cuff repair: an analysis of utility scores and cost-effectiveness. J Shoulder Elbow Surg. 2007; 16(2): 181-7.

4. Liu J, Fan L, Zhu Y, Yu H, Xu T, Li G. Comparison of clinical outcomes in all-arthroscopic versus mini-open repair of rotator cuff tears: A randomized clinical trial. Medicine (Baltimore). 2017; 96(11): e6322.

5. Williams G Jr, Kraeutler MJ, Zmistowski B, Fenlin JM Jr. No difference in postoperative pain after arthroscopic versus open rotator cuff repair. Clin Orthop Relat Res. 2014; 472: 2759-65.

6. Ji X, Bi C, Wang F, Wang Q. Arthroscopic versus mini-open rotator cuff repair: an up-to-date meta-analysis of randomized controlled trials. Arthroscopy. 2015; 31(1): 118-24.

7. Greenall G, Carr A, Beard D, Rees J, Rangan A, Merritt N, et al. Systematic review of the surgical management of rotator cuff repair with an augmentative patch: a feasibility study protocol. Syst Rev. 2018; 7(1): 187. doi: 10.1186/s13643-018-0851-1.

8. Hui YJ, Teo AQ, Sharma S, Tan BH, Kumar VP. Immediate costs of mini-open versus arthroscopic rotator cuff repair in an Asian population. J Orthop Surg (Hong Kong). 2017; 25(1): 2309499016684496.

9. Tashjian RZ, Belisle J, Baran S, Granger EK, Nelson RE, Burks RT, et al. Factors influencing direct clinical costs of outpatient arthroscopic rotator cuff repair surgery. J Shoulder Elbow Surg. 2018; 27(2): 237-41.

10. Churchill RS, Ghorai JK. Total cost and operating room time comparison of rotator cuff repair techniques at low, intermediate, and high volume centers: mini-open versus all-arthroscopic. J Shoulder Elbow Surg. 2010; 19(5): 716-21.
11. Adla DN, Rowsell M, Pandey R. Cost-effectiveness of open versus arthroscopic rotator cuff repair. J Shoulder Elbow Surg. 2010; 19(2): 258-61.

12. Mohtadi NG, Hollinshead RM, Sasyniuk TM, Fletcher JA, Chan DS, Li FX. A randomized clinical trial comparing open to arthroscopic acromioplasty with mini-open rotator cuff repair for full-thickness rotator cuff tears: disease-specific quality of life outcome at an average 2-year follow-up. Am J Sports Med. 2008; 36(6): 1043-51.

13. Colegate-Stone T, Allom R, Tavakkolizadeh A, Sinha J. An analysis of outcome of arthroscopic versus mini-open rotator cuff repair using subjective and objective scoring tools. Knee Surg Sports Traumatol Arthrosc. 2009; 17(6): 691-4.

14. Barra-López ME. El test de Constant-Murley. Una revisión de sus características. Rehabilitación. 2007; 41: 228-35.

15. Pérez JCV. Costos Unitarios por Nivel de Atención Médica que regirán para el ejercicio 2017. México: Diario Oficial de la Federación México; 2017. pp. 1-7.

16. Reglamento de la Ley General de Salud en Materia de Investigación; última reforma publicada en el DOF 02-04-2014. [Consultado 11/01/2020] Disponible en: http://www.diputados.gob.mx/ LeyesBiblio/regley/Reg_LGS_MIS.pdf

17. Carr AJ, Cooper CD, Campbell MK, Rees JL, Moser J, Beard DJ, et al. Clinical effectiveness and cost-effectiveness of open and arthroscopic rotator cuff repair [the UK Rotator Cuff Surgery (UKUFF) randomised trial]. Health Technol Assess. 2015; 19(80): 1-218.

18. Bond EC, Hunt L, Brick MJ, Leigh WB, Maher A, Young SW, et al. Arthroscopic, open and mini-open approach for rotator cuff repair: no difference in pain or function at 24 months. ANZ J Surg. 2018; 88(12): $50-5$.

19. Huang R, Wang S, Wang Y, Qin X, Sun Y. Systematic review of allarthroscopic versus mini-open repair of rotator cuff tears: a metaanalysis. Sci Rep. 2016; 6: 22857.

20. Zhang Z, Gu B, Zhu W, Zhu L, Li Q. Arthroscopic versus mini-open rotator cuff repair: a prospective, randomized study with 24-month follow-up. Eur J Orthop Surg Traumatol. 2014; 24(6): 845-50.

21. Day MA, Westermann RW, Bedard NA, Glass NA, Wolf BR. Trends associated with open versus arthroscopic rotator cuff repair. HSS J. 2019; 15(2): 133-6.

22. Murphy J, Gray A, Cooper C, Cooper D, Ramsay C, Carr A. Costs, quality of life and cost-effectiveness of arthroscopic and open repair for rotator cuff tears: an economic evaluation alongside the UKUFF trial. Bone Joint J. 2016; 98-B(12): 1648-55.

23. Osti L, Papalia R, Paganelli M, Denaro E, Maffulli N. Arthroscopic vs mini-open rotator cuff repair. A quality of life impairment study. Int Orthop. 2010; 34(3): 389-94.

24. Karaman O, Karakus O, Saygi B. A comparison of full arthroscopic and arthroscopic-assisted mini-open repair methods in rotator cuff tears 1-3 cm in size. J Orthop. 2018; 15(3): 894-8.

25. Aparicio JL. Lesiones del manguito rotador del hombro. Tratamiento: artroscopía más abordaje mínimo (mini open repair). Rev Asoc Arg Ortop y Traumatol. 1998; 63: 35-40.

Conflictos de intereses: Los autores no tienen conflicto de intereses que declarar ni denunciar.

Financiamiento: La presente investigación no ha recibido ninguna beca específica de agencias de los sectores público, comercial o sin ánimo de lucro. 